\title{
Letramento em Saúde: Avaliação de pacientes em terapia renal substitutiva
}

\author{
Késia Tomasi da Rocha ${ }^{1}$, Ana Elizabeth Figueiredo ${ }^{2}$ \\ ${ }^{1}$ Hospital São Lucas. Pontifícia Universidade Católica do Rio Grande do Sul. Brasil \\ ${ }^{2}$ Escola de Ciências da Saúde e da Vida da Pontifícia Universidade Católica do Rio Grande do Sul. Brasil
}

\section{Resumo}

Introdução: considerando a prevalência de pacientes em terapia renal substitutiva, torna-se extremamente revelevante a avaliação da capacidade de entendimento e aplicacação das orientações que esses indíviduos recebem nos serviços de saúde.

Objetivo: estudar o impacto do nível de letramento em saúde de pacientes submetidos à hemodiálise, diálise peritoneal e transplante renal, associado aos aspectos cognitivos, adesão medicamentosa e qualidade de vida.

Material e Método: estudo transversal, realizado com indivíduos acima de 18 anos, submetidos a algum tipo de terapia renal substitutiva, há três meses consecutivos. Foram aplicadas as versões brasileiras dos instrumentos: Short Assessment of Health Literacy for Portuguese-speaking Adults-18, Mini Exame do Estado Mental, 36-Item Short Form e a escala de Morisky, além de um questionário sobre dados clínicos e sociodemográficos.

Resultados: foram avaliados 138 pacientes, sendo 50 $(36,2)$ submetidos à hemodiálise, $23(16,7 \%)$ a diálise peritoneal e $65(47,1 \%)$ ao transplante renal. A média de idade foi $52,0 \pm 15,5$ anos e $58,0 \%$ do sexo masculino. Os pacientes com letramento inadequado $(51,4 \%)$ tinham renda igual ou inferior a um salário mínimo $(P=0,002)$ e ensino fundamental completo ou inferior $(P<0,001)$. Os indivíduos com letramento ade-

\section{Correspondencia:}

Késia Tomasi da Rocha

E-mail: trkesia@gmail.com

Como citar este artículo:

Enferm Nefrol. 2019 Oct-Dic;22(4):388-97

\section{Alfabetización en salud: valoración de pacientes en tratamiento renal sustitutivo}

\section{Resumen}

Introducción: Considerando la prevalencia de pacientes en tratamiento renal sustitutivo, es extremamente relevante la valoración de la capacidad de comprensión y aplicación de las orientaciones que esos individuos reciben en los servicios de salud.

Objetivo: estudiar el impacto del nivel de alfabetización en salud de pacientes sometidos a hemodiálisis, diálisis peritoneal y trasplante renal, asociado a los aspectos cognitivos, adhesión a la medicación y calidad de vida.

Material y Método: Estudio transversal, realizado con individuos mayores de 18 años, sometidos a algún tipo de tratamiento renal sustitutivo, durante al menos tres meses consecutivos. Fueron aplicadas las versiones brasileñas de los instrumentos: Short Assessment of Health Literacy for Portuguese-speaking Adults-18, 
"Mini Exame do Estado Mental, 36-Item Short Form y la Escala de Morisky, así como un cuestionario sobre datos clínicos y sociodemográficos.

Resultados: se valoraron 138 pacientes, 50 (36,2\%) sometidos a hemodiálisis, $23(16,7 \%)$ a diálisis peritoneal y $65(47,1 \%)$ trasplantados renales. La media de edad fue de $52,0 \pm 15,5$ años y $58,0 \%$ del sexo masculino. Los pacientes con alfabetización en salud inadecuada $(51,4 \%)$ tenían salario igual o inferior a un salario mínimo $(P=0,002)$ y educación básica completa o inferior $(P=0,001)$. Los individuos con alfabetización adecuada presentaban educación secundaria incompleta o mayor escolaridad, salario igual o superior a cinco salarios mínimos y mayor puntuación en el dominio de aspectos emocionales referentes a calidad de vida $(P=0,052)$.

Conclusiones: Nuestros resultados sugieren que el nivel de literacia en salud está asociado con el salario y escolaridad en los pacientes que realizan tratamiento renal sustitutivo.

PALABRAS CLAVE: alfabetización en Salud; educación para la salud; enfermedad renal crónica; terapia de reemplazo renal; comunicación en salud.

\section{Health literacy: assessment of patients in renal replacement therapy}

\section{Abstract}

Introduction: Considering the prevalence of patients on renal replacement therapy, it is extremely revealing to evaluate the ability to understand and apply the guidelines that these individuals receive in health services. Objective: To study the impact of the health literacy level of patients on hemodialysis, peritoneal dialysis and kidney transplantation, associated with cognitive aspects, therapeutic adherence and quality of life.

Material and Method: Cross-sectional study conducted with individuals over 18 years old, undergoing some type of renal replacement therapy, for three consecutive months. The Brazilian versions of the instruments were applied: Short Assessment of Health Literacy for Portuguese-speaking Adults-18, Mini Mental State Examination, 36-Item Short Form and the Morisky scale, as well as a questionnaire on clinical and sociodemographic data.
Results: 138 patients were evaluated, $50(36.2 \%)$ on hemodialysis treatment, 23 ( $16.7 \%$ ) peritoneal dialysis and $65(47.1 \%)$ renal transplantation. The average age was $52.0 \pm 15.5$ years and $58.0 \%$ male. Patients with inadequate literacy $(51.4 \%)$ had an income equal to or less than the minimum salary $(P=0.002)$ and completed elementary school or lower $(P<0.001)$. Individuals with adequate literacy had incomplete high school or higher education, income equal to or higher than five minimum salaries and higher score in the 'emotional aspects related to quality of life' domain ( $P=0.052)$.

Conclusions: Our results suggest that the level of literacy is associated with income and education in patients undergoing renal function replacement.

KEYWORDS: health literacy; health education; chronic kidney disease; renal replacement therapy; health communication.

\section{Introdução}

A incidência e prevalência da doença renal crônica terminal (DRCT) variam globalmente e estima-se que, a cada um milhão de pessoas, 280 indivíduos estejam em hemodiálise (HD) ou diálise peritoneal (DP) e 65 tenham um transplante renal (TxR) em funcionamento ${ }^{1}$.

Cada um dos tipos de terapia renal substitutiva (TRS) requerirá habilidades distintas dos indivíduos que as realizam. Na HD, por exemplo, é necessário que o paciente siga uma dieta mais rígida devido a frequência da terapia (em geral, apenas três vezes por semana), além dos cuidados com o acesso vascular². Enquanto a DP, requer que o paciente ou cuidador tenham capacidade de compreender as etapas do método e realizá-las no domicílio, além de saber reconhecer sinais de peritonite e outras complicações ${ }^{3}$. Já no TxR, a adesão do paciente aos medicamentos prescritos, bem como a aplicação dos cuidados recebidos referentes ao seu estado imunossupresso são a chave para o êxito da terapia 4 .

Assim, é necessário que os pacientes tenham condições de entender, significar e aplicar as instruções que recebem, estando tais aspectos contemplados no letramento em saúde (LS), o qual consiste na capacidade de obter, processar e compreender as informações e os serviços básicos de saúde necessários para a tomada de decisão $0^{5}$. É extremamente relevante de ser mensurado 
na população em TRS, porque apresenta um potencial de impacto em diferentes desfechos clínicos ${ }^{6,7}$.

0 estudo de Finkelstein, realizado com pacientes nos estágios 3-5 em tratamento conservador, mostrou 0 percentual de conhecimento sobre as modalidades de TRS, sendo que na DRCT, apenas 54\% apresentava informações sobre HD, 53\% quando referente a DP e $79 \%$ sobre o $\mathrm{TxR}^{8}$. Além disso, ficou evidente que apenas a consulta com o nefrologista não garante que o paciente saiba as opções de substituição da função renal ${ }^{8}$.

Em uma outra pesquisa, realizada com 480 pacientes em HD provenientes de 77 clínicas de diálise, foi encontrada a prevalência de LS inadequado em $32 \%$ dos participantes (segundo o instrumento Rapid Estimate of Adult Literacy of Medicine - REALM), estando mais frequentemente associado com o sexo masculino, nãobrancos e menor escolarização 0 risco de óbito foi aumentado em 54\% (hazard ratio 1,54; IC 95\% 1,01 a $2,36)$ nos pacientes com letramento limitado 9.

Um estudo realizado com pacientes transplantados renais mostrou níveis baixos de LS pela ferramenta Health Literacy Questionnaire (HLQ), a qual mensura, dentre outros aspectos, o gerenciamento ativo da saúde e avaliação crítica de informações ${ }^{10}$. Além disso, recentemente, tem sido investigado o impacto da seleção de pacientes com LS adequado no transplante, considerando que a prevalência de letramento limitado é menor em receptores de TxR do que em pacientes que iniciam diálise ${ }^{11}$.

Juntamente com a verificação do $L S$, é recomendada a avaliação dos aspectos cognitivos dos pacientes com DRC, devido a presença de múltiplas comorbidades e alterações metabólicas que impactam indiretamente no planejamento de ações e falhas na memória ${ }^{12}$. Além disso, outras pesquisas buscaram verificar a associação da adesão ao tratamento farmacológico e qualidade de vida com o nível de LS, demonstrando que tais variáveis são importantes de serem avaliadas quando considerado o contexto dos pacientes em TRS10,13.

Assim, dada a crescente incidência e complexidade do tratamento da DRCT e a influência do LS na participação ativa do paciente em sua terapia, o objetivo desta pesquisa é estudar o impacto do nível de LS, associado aos aspectos cognitivos, na adesão ao tratamento medicamentoso e qualidade de vida ( $Q V)$ dos pacientes submetidos à TRS.

\section{Material e Método}

Estudo transversal, realizado no período de junho à outubro/2018, em que foram incluídos pacientes maiores de 18 anos, submetidos a HD, DP e TxR, por mais de três meses consecutivos e em acompanhamento no Serviço de Nefrologia de um Hospital Universitário de Porto Alegre - Rio Grande do Sul. Foram excluídos os pacientes com TxR pré-emptivo, declínio cognitivo grave (pontuação <10 no Mini Exame do Estado Mental) e/ ou deficiências físicas (diminuição severa da acuidade visual e/ou auditiva) que impossibilitasse a participação do sujeito neste estudo.

Após o processo de consentimento, foram aplicados os seguintes instrumentos: formulário de dados sociodemográficos e clínicos, Escala de Morisky, MiniExame do Estado Mental (MEEM), Short Assessment of Health Literacy for Portuguese-speaking Adults -18 (SAHLPA-18) e o 36-Item Short Form (SF-36).

A adesão medicamentosa foi medida pela Escala de Morisky, a qual é composta por quatro perguntas fechadas, sendo as respostas pontuadas em sim (em relação à adesão, atribuído o valor de zero) ou não (em relação a não adesão, atribuído o valor de um) ${ }^{14}$. Escore zero indica máxima adesão, de um a dois, moderada e de três a quatro, baixa adesão ${ }^{14}$.

Os aspectos cognitivos foram avaliados pelo instrumento MEEM, que considera a cognição global: habilidades visuoespaciais, função executiva, linguagem, memória, atenção e orientação, cálculo e abstração, sendo o escore global obtido pelo somatório dos itens, tendo como valor máximo 30 pontos $^{15}$. Classifica-se em: $<24$ pontos - declínio cognitivo; entre 23 e 21 - declínio leve; entre 20 e 11 - declínio moderado e menor que 10 - declínio grave ${ }^{15}$. Embora muitos autores utilizem os anos de escolaridade para definir os pontos de corte da ferramenta MEEM, salienta-se que, nesse estudo, tal instrumento foi utilizado apenas com o objetivo de avaliar o perfil da amostra e excluir os pacientes com declínio cognitivo grave ${ }^{16}$.

0 LS foi mensurado pelo instrumento SAHLPA-18, que avalia habilidades de pronúncia e compreensão de termos médicos comuns ${ }^{17}$. Esta ferramenta é composta por 18 cartões, em que há um termo principal em negrito que 0 indivíduo deve pronunciar em voz alta e duas palavras abaixo de associação, as quais o participante deve escolher a palavra que melhor se relacione com o termo lido por ele ${ }^{17}$. 0 item é considerado correto 
apenas quando o participante acerta a pronúncia e a associação. Cada item correto recebe um ponto e o escore total é obtido pela soma dos itens, variando de 0 a 18, em que o escore de 0 a 14 sugere alfabetismo em saúde inadequado ${ }^{17}$.

A avaliação da $Q V$, deu-se pelo questionário SF-36, o qual é validado para o Português do Brasil ${ }^{18}$. Divide-se em oito dimensões, sendo elas: capacidade funcional, aspectos físicos, emocionais, dor, estado geral de saúde, vitalidade, aspectos sociais, saúde mental e mais uma questão de avaliação comparativa entre as condições de saúde atual e as de um ano atrás, que é de extrema importância para 0 conhecimento da doença do paciente ${ }^{18}$. Apresenta um escore final de 0 a 100, no qual zero corresponde ao pior estado geral de saúde e 100 ao melhor ${ }^{18}$.

0 cálculo do tamanho da amostra foi realizado no programa WinPEPI (Programs for Epidemiologists for Windows) versão 11.43 e foi baseado no estudo de Dodson e colaboradores ${ }^{13}$. Considerando um nível de significância de $5 \%$, poder de $95 \%$, prevalência estimada de baixo nível de LS em 57\%, obteve-se um total mínimo de 108 pacientes.

Os dados foram analisados com Statistical Package for the Social Sciences 21.0 e a normalidade da distribuição dos dados quantitativos foi verificada com o teste Kolmogorov Smirnov (KS).

$\mathrm{Na}$ fase descritiva, as variáveis quantitativas foram apresentadas por medidas de tendência central (média e mediana) e dispersão (desvio-padrão e intervalo interquartil) e, as qualitativas, por meio de frequência absoluta e relativa. $\mathrm{Na}$ fase analítica, baseada nos resultados do teste KS, a comparação entre as variáveis quantitativas com distribuição não-normal foi realizada através do teste Mann Whitney (domínios do questionário SF-36) e, nas demais, foi aplicado o teste T de Student. A associação entre variáveis qualitativas foi verificada com os testes Quiquadrado de Pearson e Exato de Fischer. 0 teste de regressão de Poisson foi utilizado para a análise multivariada. Foi considerado significativo um $\mathrm{P}<0,05$.
Buscando contemplar os princípios legais e éticos, de acordo com as Normas e Diretrizes Regulamentadoras da pesquisa em seres humanos, do Conselho Nacional de Saúde (resolução 466/2012), este estudo foi aprovado pelo Comitê de Ética em Pesquisa da Pontifícia Universidade Católica do Rio Grande do Sul. Todos os participantes passaram pelo processo de consentimento, assinando o Termo de Consentimento Livre e Esclarecido.

\section{Resultados}

Aproximadamente 630 pacientes transplantados renais acompanhavam regularmente no ambulatório em que o estudo foi realizado, dos quais 65 participaram do presente estudo. Dos sujeitos em diálise, havia cerca de 88 em HD e 46 submetidos a DP, sendo que 50 e 23 pacientes participaram dessa pesquisa, respectivamente (Figura 1).

Foram avaliados 138 pacientes com média de idade de $52,0 \pm 15,5$ anos (intervalo 19 a 84 anos), a maioria com menos de 65 anos $(76,1 \%)$, do gênero masculino $(58,0 \%)$ e, mais frequentemente, com ensino médio completo $(42,8 \%)$ e renda de dois a cinco salários mínimos (49,3\%) (Tabela 1).

A mediana dos dias em TRS de todos os participantes foi 995 (126 à 9.369). Aproximadamente 39,1\% $(n=54)$ dos transplantados renais realizavam $H D$ antes do TxR e, quando estratificado por anos ( $\leq 1$ ano, $>1$ até $\leq 5$ anos e $>5$ anos), tanto os dias de exposição à doença, quanto os dias em diálise, não apresentaram significância estatística ( $P=0,40$ e $P=0,89$, respectivamente).

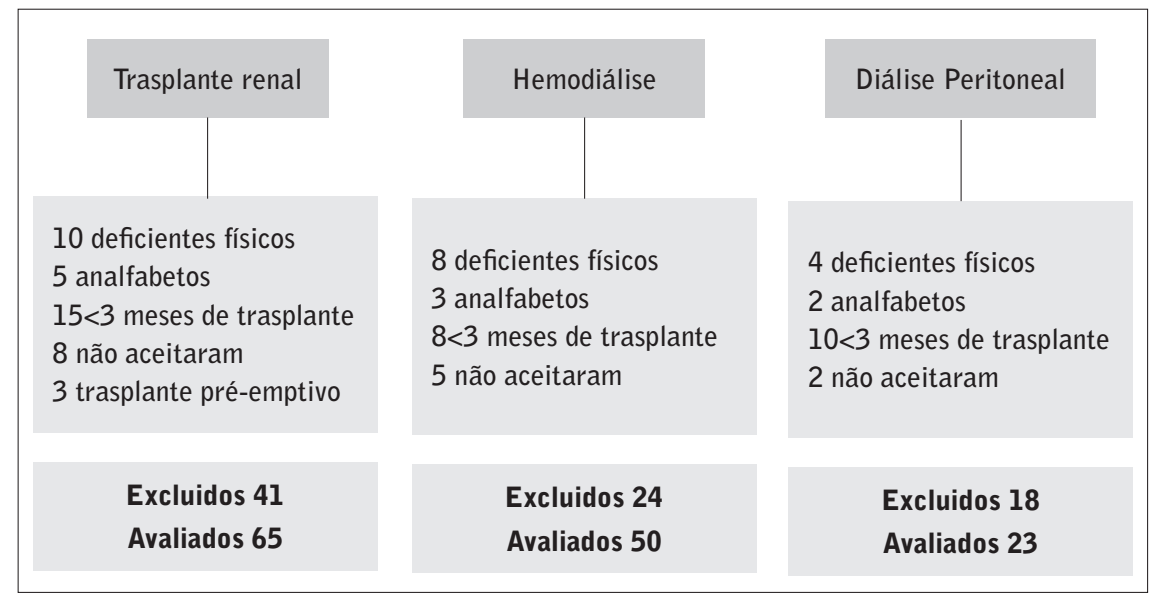

Figura 1. Descrição da amostra: participantes excluídos e avaliados no estudio. Fonte: dados da pesquisa (2018). 
Tabela 1. Características da amostra de pacientes submetidos à terapia renal substitutiva atendidos no Serviço de Nefrologia de um Hospital Universitário de Porto Alegre - RS ( $\mathrm{N}=138)$.

\begin{tabular}{|c|c|}
\hline Variáveis & N (\%) \\
\hline \multicolumn{2}{|l|}{ Sociodemográficas } \\
\hline $\begin{array}{l}\text { Idade em anos (média } \pm D P \text { ) } \\
\text { Gênero masculino }\end{array}$ & $\begin{array}{l}52,0 \pm 15,5 \\
80(58,0)\end{array}$ \\
\hline \multicolumn{2}{|l|}{ Escolaridade } \\
\hline $\begin{array}{l}\text { Fundamental incompleto } \\
\text { Fundamental completo } \\
\text { Médio incompleto } \\
\text { Médio completo } \\
\text { Superior }\end{array}$ & $\begin{array}{l}23(16,7) \\
28(20,3) \\
11(8,0) \\
59(42,8) \\
17(12,3)\end{array}$ \\
\hline \multicolumn{2}{|l|}{ Renda mensal em salários mínimos } \\
\hline $\begin{array}{l}\leq \mathrm{Um} \\
2 \text { a } 5 \\
>5\end{array}$ & $\begin{array}{l}46(33,3) \\
68(49,3) \\
24(17,4)\end{array}$ \\
\hline \multicolumn{2}{|l|}{ Dados clínicos } \\
\hline \multicolumn{2}{|l|}{ Doença de base } \\
\hline $\begin{array}{l}\text { Diabete Mellitus } \\
\text { Hipertensão arterial sistêmica }\end{array}$ & $\begin{array}{l}21(15,2) \\
31(22,5)\end{array}$ \\
\hline \multicolumn{2}{|l|}{ Polifarmácia } \\
\hline Cinco ou mais medicações/dia & $110(79,7)$ \\
\hline *Presença de cuidador & $25(18,1)$ \\
\hline
\end{tabular}

*A presença de cuidador foi avaliada apenas como caracterização do perfil da amostra, independente do desfecho de saúde dos participantes. Salário mínimo: $\mathrm{R} \$ 954,00$. Fonte: dados dos próprios pesquisadores (2018).

Quanto ao tempo de aplicação do instrumento SAHLPA, a média em minutos foi de $4,1 \pm 1,5$. Para os pacientes com o letramento inadequado, 0 tempo de reposta dessa ferramenta foi $4,70 \pm 1,64$ minutos, estando significativamente associado a esta classificação $(P<0,001)$. A média do escore deste instrumento foi $13,7 \pm 3,8$ pontos e a pronúncia foi o aspecto que os pacientes mais acertaram $45,7 \%(n=63)$, enquanto que, $28,3 \%(n=39)$ realizaram corretamente a associação e $26,1 \%(n=36)$ tiveram acertos iguais. As palavras que os participantes menos acertaram foram "icterícia" e "incesto" (Tabela 2).

A maioria dos pacientes classificados como inadequados em relação ao LS, tinham renda igual ou inferior a um salário mínimo e ensino fundamental completo ou inferior, enquanto que, os indivíduos com letramento adequado, apresentavam, mais frequentemente, ensino médio incompleto ou maior escolaridade e renda de cinco salários mínimos ou mais (Tabela 3).

Quando comparadas as diferentes modalidades de TRS, a DP foi a que apresentou maior nível de letramento
Tabela 2. Descrição de acertos dos participantes, por palavra, do instrumento Short Assessment of Health Literacy for Portuguesespeaking Adults (SAHLPA - 18).

\begin{tabular}{|l|l|c|c|}
\hline $\begin{array}{l}\text { Palavras do } \\
\text { instrumento SAHLPA }\end{array}$ & $\begin{array}{l}\text { Pronúncia } \\
\text { N } \%)\end{array}$ & $\begin{array}{c}\text { Associação } \\
\text { N }(\%)\end{array}$ & $\begin{array}{c}\text { Total } \\
\text { N }(\%)\end{array}$ \\
\hline Osteoporose & $102(73,9)$ & $135(97,8)$ & $101(73,2)$ \\
\hline Papanicolau & $113(81,9)$ & $116(84,1)$ & $97(70,3)$ \\
\hline Aborto & $136(98,6)$ & $131(94,9)$ & $129(93,5)$ \\
\hline Hemorroida & $118(85,5)$ & $127(92,0)$ & $113(81,9)$ \\
\hline Anormal & $135(97,8)$ & $119(86,2)$ & $117(84,8)$ \\
\hline Menstrual & $131(94,9)$ & $131(94,9)$ & $126(91,3)$ \\
\hline Comportamento & $135(97,8)$ & $124(89,9)$ & $123(89,1)$ \\
\hline Convulsão & $129(93,5)$ & $135(97,8)$ & $126(91,3)$ \\
\hline Retal & $135(97,8)$ & $119(86,2)$ & $117(84,8)$ \\
\hline Apêndice & $101(73,2)$ & $119(86,2)$ & $93(67,4)$ \\
\hline Artrite & $125(90,6)$ & $110(79,7)$ & $103(74,6)$ \\
\hline Cafeína & $134(97,1)$ & $127(92,0)$ & $124(89,9)$ \\
\hline Colite & $128(92,8)$ & $94(68,1)$ & $91(65,9)$ \\
\hline Vesícula biliar & $115(83,3)$ & $114(82,6)$ & $101(73,2)$ \\
\hline Icterícia & $75(54,3)$ & $87(63,0)$ & $54(39,1)$ \\
\hline Próstata & $114(82,6)$ & $112(81,2)$ & $99(71,7)$ \\
\hline Incesto & $98(71,0)$ & $91(65,9)$ & $69(50,0)$ \\
\hline Testículo & $129(93,5)$ & $99(71,7)$ & $94(68,1)$ \\
\hline
\end{tabular}

Fonte: dados dos próprios pesquisadores (2018).

adequado, com 56,5\% (13), seguida pela HD 54,0\% (27) e, por último, os transplantados renais com $41,5 \%$ (27). As características referentes a adesão medicamentosa, rastreamento cognitivo e QV estão descritas na tabela 4.

Em relação a terapia medicamentosa, apresentaram adesão máxima $43 \%(n=10)$ dos pacientes submetidos a DP, $57 \%(n=27)$ dos indivíduos em HD e $69,2 \%$ $(n=45)$ dos transplantados renais, não sendo encontrada associação significativa com o nível de letramento.

Quando avaliada a QV, tanto o domínio referente à limitação por aspectos físicos quanto 0 de aspectos emocionais do questionário SF-36 apresentaram distribuições não-paramétricas. A mediana e intervalo interquartil para os pacientes com letramento adequado foram 25,0 (0,0-75,0) e 66,7 (33,3-100,0) e nos sujeitos com letramento inadequado foram $25,0(0,0$ $75,0)$ e $33,3(0-100,0)$, com $\mathrm{P}=0,869$ e $\mathrm{P}=0,044$, respectivamente - teste Mann Whitney.

Considerando que renda e escolaridade apresentaram associação estatisticamente significativa com letramento, foi realizada uma análise multivariada ajustada para 
Tabela 3. Características sociodemográficas de acordo com o letramento em saúde de pacientes atendidos no Serviço de Nefrologia de um Hospital Universitário de Porto Alegre - RS ( $N=138)$.

\begin{tabular}{|c|c|c|c|}
\hline \multirow[b]{2}{*}{$\begin{array}{l}\text { Características } \\
\text { sociodemográficas }\end{array}$} & \multicolumn{2}{|c|}{ Letramento } & \multirow[b]{2}{*}{$\mathbf{P}$} \\
\hline & $\begin{array}{l}\text { Adequado } \\
\qquad \begin{array}{c}N=67 \\
N(\%)\end{array}\end{array}$ & $\begin{array}{c}\text { Inadequado } \\
\qquad \begin{array}{c}\mathrm{N}=71 \\
\mathrm{~N}(\%)\end{array}\end{array}$ & \\
\hline Idade em anos (média $\pm D P$ ) & $49,5 \pm 14,3$ & $54,4 \pm 16,3$ & 0,060 \\
\hline \multicolumn{4}{|l|}{ Faixa etária em anos } \\
\hline $\begin{array}{l}<65 \\
\geq 65\end{array}$ & $\begin{array}{l}54(80,6) \\
13(19,4)\end{array}$ & $\begin{array}{l}51(71,8) \\
20(28,2)\end{array}$ & 0,228 \\
\hline \multicolumn{4}{|l|}{ Gênero } \\
\hline $\begin{array}{l}\text { Masculino } \\
\text { Feminino }\end{array}$ & $\begin{array}{l}37(55,2) \\
30(44,8)\end{array}$ & $\begin{array}{l}43(60,6) \\
28(39,4)\end{array}$ & 0,525 \\
\hline \multicolumn{4}{|l|}{ Escolaridade } \\
\hline $\begin{array}{l}\text { Fundamental incompleto } \\
\text { Fundamental completo } \\
\text { Médio incompleto } \\
\text { Médio completo } \\
\text { Superior }\end{array}$ & $\begin{array}{c}3(4,5) \\
5(7,5) \\
9(13,4) \\
36(53,7) \\
14(20,9)\end{array}$ & $\begin{aligned} 20 & (28,2) \\
23 & (32,4) \\
2 & (2,8) \\
23 & (32,4) \\
3 & (4,2)\end{aligned}$ & $<0,001$ \\
\hline \multicolumn{4}{|l|}{$\begin{array}{l}\text { Renda mensal } \\
\text { em salários mínimos }\end{array}$} \\
\hline $\begin{array}{l}\leq U m \\
2 \text { a } 5 \\
>5\end{array}$ & $\begin{array}{l}16(23,9) \\
32(47,8) \\
19(28,4)\end{array}$ & $\begin{array}{c}30(42,3) \\
36(50,7) \\
5(7,0)\end{array}$ & 0,002 \\
\hline
\end{tabular}

Teste T de Student para comparação de médias e Teste Qui-quadrado para associação entre medidas categóricas. Salário mínimo: R \$954,00. Fonte: dados dos próprios pesquisadores (2018).

essas duas variáveis, na qual se encontrou que o declínio cognitivo não se manteve associado com LS $(P>0,05)$ (Tabela 5).

\section{Discussão}

Esta pesquisa objetivou estudar o impacto do nível de LS, associado aos aspectos cognitivos, na adesão ao tratamento medicamentoso e QV dos pacientes submetidos à TRS. Destaca-se a relevância deste por haver um número pequeno de publicações que avaliam o LS, sendo ainda mais limitado quando se trata de pacientes portadores de $\mathrm{DRCT}^{7}$.

Escolaridade e renda tiveram associação com a inadequação do LS na maior parte dos pacientes deste estudo, sendo isto apontado pela literatura independente da ferramenta que se utilize. Cavanaugh e cols, utilizando o REALM, encontraram $30 \%$ de LS limitado em pacientes submetidos a HD $(n=480)$, estando isto associado ao ensino fundamental completo ou inferior
Tabela 4. Tipo de terapia renal substitutiva, adesão medicamentosa, qualidade de vida e aspectos cognitivos de acordo com o letramento em saúde de pacientes atendidos no Serviço de Nefrologia de um Hospital Universitário de Porto Alegre - RS ( $\mathrm{N}=138$ ).

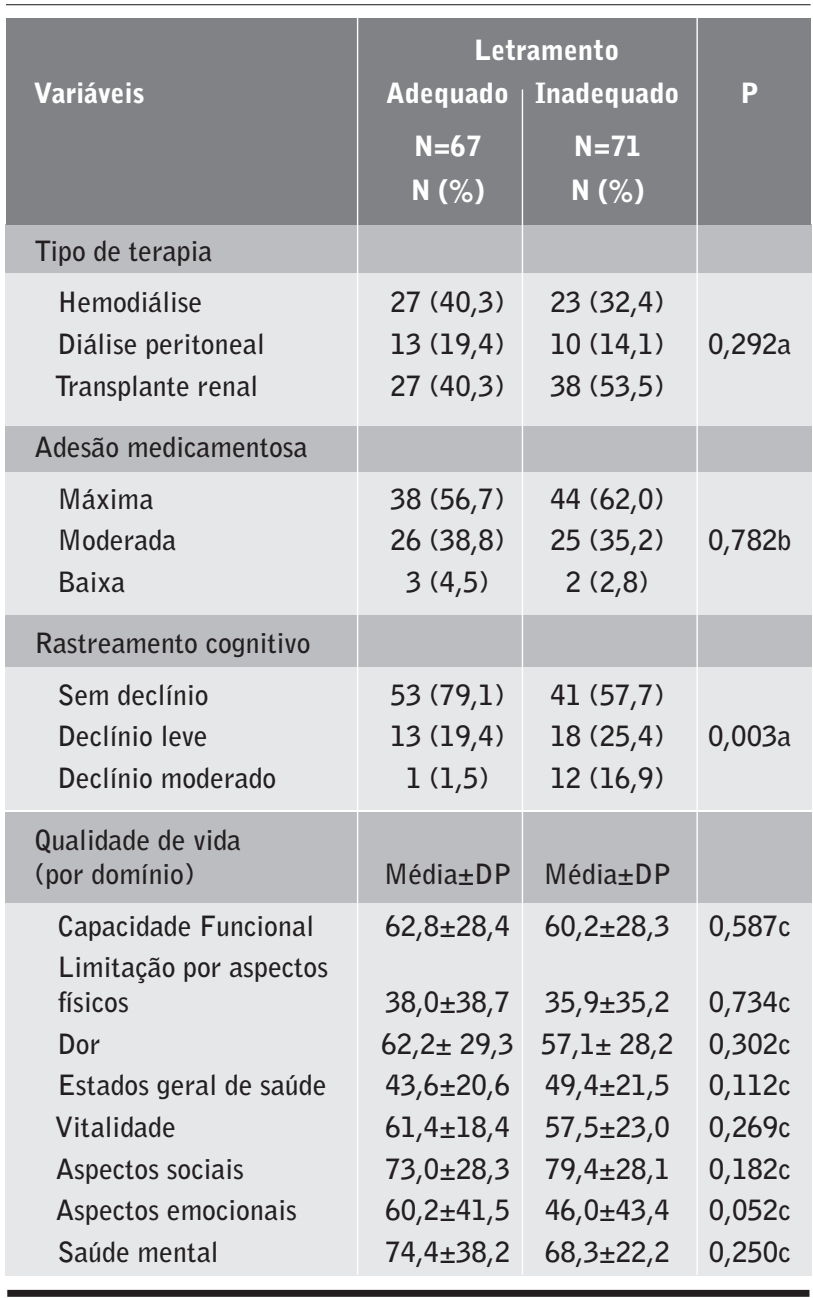

a: Teste Qui-quadrado de Pearson; b: Teste Exato de Fischer; $\mathbf{c}$ : Teste T de Student. Fonte: dados dos próprios pesquisadores (2018).

Tabela 5. Análise multivariada do rastreamento cognitivo de pacientes atendidos no Serviço de Nefrologia de um Hospital Universitário de Porto Alegre - RS ( $N=138)$.

\begin{tabular}{lcccc}
\hline & \multicolumn{4}{c}{ Intervalo de confiança 95\% } \\
Variáveis & RP & Inferior & Superior & P \\
\hline Declínio cognitivo & & & & \\
\hline Moderado & 1,474 & 0,994 & 2,185 & 0,053 \\
Leve & 1,267 & 0,879 & 1,826 & 0,204 \\
Ausente & 1 & & & \\
\hline
\end{tabular}

Regressão de Poisson ajustada para renda e escolaridade. Fonte: dados dos próprios pesquisadores (2018). 
$(P<0,001)^{9}$. Este achado é endossado pelo estudo de Green, realizado nos Estados Unidos com 260 pacientes em HD, que também encontrou associação do letramento inadequado com a baixa educação formal e renda ${ }^{19}$.

Quando a ferramenta utilizada foi 0 Short Test of Functional Health Literacy in Adults (S-TOFHLA), resultados semelhantes foram encontrados por Brice e colaboradores ${ }^{20}$, em que o LS marginal e inadequado foi constatado em $46 \%$ ( $n=107$ ) dos pacientes em HD, enquanto que, no estudo realizado por Adeseun e cols ${ }^{21}$, a prevalência foi de $21 \%$ de letramento inadequado e as variáveis escolaridade e renda apresentaram significância estatística $(P<0,001)$, bem como a modalidade de diálise $(P=0,04)$.

Em relação ao nível de letramento na DP, no estudo de Jain, $19 \%$ dos pacientes apresentaram letramento inadequado, sendo um pouco acima do encontrado nesta pesquisa ${ }^{22}$. Educação formal apresentou relação com este achado $(P=0,02)^{22}$. Infelizmente, os poucos estudos que avaliam a população em DP, apresentam os seus resultados conjuntamente com a HD, dificultando a comparação deste estudo com outros resultados ${ }^{13,23}$.

Referente aos transplantados renais, o estudo de Demian e colaboradores, avaliou 96 pacientes, com a ferramenta Health Literacy Questionnaire (HLQ) ${ }^{10}$. Nessa pesquisa, houve associação da baixa adesão medicamentosa com o nível inadequado de $\mathrm{LS}(\mathrm{P}=0,004)^{10}$. Outro estudo, fomenta que o letramento pode ser utilizado como um preditor para desfechos dos transplantados renais, sugerindo que os profissionais devem considerar a sua avaliação ao conversar com os pacientes sobre $0 T_{x} R^{24}$. Ainda, tem-se a hipótese de que, quando comparado o nível de LS entre receptores de rins com doador vivo ou falecido, os receptores de doadores falecidos são mais propensos ao LS inadequado, instigando a realização de mais estudos nessa área ${ }^{25}$.

A variável cor, como componente de instrumentos de pesquisa, vem sendo discutida nos últimos anos no meio científico devido a necessidade de se implementar uma nomenclatura mais inclusiva, a saber por origem geográfica do participante ${ }^{26}$. Embora o objetivo desse estudo não seja avaliar esse aspecto, ressalta-se que um estudo multicêntrico, realizado com uma amostra significativa de pacientes em HD, apontou a cor não-branca como uma variável relacionada ao LS inadequado?.

Quanto a escolaridade, mais de dois terços dos participantes apresentavam ensino médio completo ou nível superior, porém o LS inadequado foi o mais prevalente na amostra. A relação pronúncia-significado foi desproporcional e, mesmo com escolaridade superior, a correta associação do significado com os termos pronunciados ocorreu em menor número. Tal achado reforça que, mesmo com escolaridade acima do ensino médio, a avaliação do LS não deve ser eximida, pois os pacientes podem se tornar meros repetidores de vocábulos que estão habituados a ouvir nos serviços de saúde, não influenciando em nada a sua tomada de decisão e trazendo uma falsa ideia de entendimento das informações que lhe são transmitidas.

A literatura mostra que, em algumas unidades de diálise, a avaliação do LS já foi incorporada à rotina assistencial ${ }^{19,22}$. Mesmo ciente das demandas diárias, 0 tempo e a facilidade de aplicação destas ferramentas são pontos positivos. Em nosso estudo a média do tempo para a resposta do instrumento SAHLPA foi quase 0 dobro do usual (1-2 minutos), entretanto, ainda sim, mostrou-se de fácil e rápida aplicação, considerando que existem outros instrumentos que podem levar de 12 a até 22 minutos para serem respondidos ${ }^{27}$.

Em relação a adesão ao tratamento medicamentoso, a Escala de Morisky é comumente utilizada para a população em HD, sendo as prevalências de baixa adesão de aproximadamente $47 \%$ a $56 \%$ nesta população ${ }^{28,29}$. Nos transplantados renais, encontrase uma variação maior (18,5\% a $76 \%$ ), devido a diversidade de instrumentos utilizados para avaliar a esse aspecto nessa população $0^{30,31}$.

Embora nossos achados não tenham mostrado associação do nível de LS com a adesão farmacológica, a literatura sugere esta relação especialmente com os pacientes transplantados, devido ao uso dos imunossupressores dentre outras tantas medicações ${ }^{10}$. Ressalta-se a importância de avaliar tais aspectos nos pacientes em diálise, pois é um grupo que igualmente apresenta variedade de comorbidades e polifarmácia.

Existe uma relação entre a progressão da DRC e o declínio cognitivo, em que os pacientes com $T F G<15$ $\mathrm{mL} / \mathrm{min} / 1,73 \mathrm{~m}^{2}$ apresentam déficit maior do que os sujeitos com TFG $>60 \mathrm{~mL} / \mathrm{min} / 1,73 \mathrm{~m}^{2}{ }^{32}$. Nossos dados corroboram com esse achado, visto que os pacientes com LS inadequado apresentaram declínio cognitivo moderado, segundo o MEEM. Cavanaugh e cols. também encontraram que a menor capacidade cognitiva está associada ao LS inadequado ${ }^{33}$. 
Considerando que a modalidade de TRS mais prevalente é a HD, esta população é também a mais investigada quanto ao declínio cognitivo nos pacientes que têm a sua função renal substituída ${ }^{32}$. 0 estudo de Condé e colaboradores avaliou os aspectos cognitivos em diferentes estágios da DRC e encontrou notadamente um déficit cognitivo maior nos pacientes em HD, entretanto, deve-se considerar que nesse estudo tal amostra era mais que o dobro, quando comparado aos demais grupos ${ }^{34}$.

Quanto a QV, embora não tenha apresentado significância estatística, nota-se que a média do domínio referente aos aspectos emocionais foi mais elevada nos pacientes com LS adequado, possivelmente, porque a melhor capacidade de compreensão do processo saúde-doença, bem como o seu prognóstico e tratamento auxiliam no enfretamento da DRC, fazendo com que os pacientes tenham menos sintomas de depressão e ansiedade. Dodson e colaboradores também encontraram menos limitações de ordem emocional nos pacientes em diálise com LS adequado ${ }^{13}$.

Tem-se como limitações do presente estudo: a verificação do nível de LS dos pacientes submetidos a TRS em apenas uma instituição e a carência de instrumentos que avaliem o LS especificamente em doentes renais crônicos.

\section{Conclusões}

0 letramento inadequado foi encontrado na maioria dos participantes dessa pesquisa, estando a renda (igual ou inferior a um salário mínimo) e escolaridade (ensino fundamental completo ou inferior) associadas à esse achado. Já nos participantes com LS adequado, o domínio dos aspectos emocionais da QV se mostrou superior, bem como a renda acima de cinco salários mínimos e a escolaridade igual ou superior do ensino médio incompleto.

Assim, sugere-se que os profissionais de saúde se utilizem da avaliação do LS como um aliado à assistência, bem como uma ferramenta para a elaboração de um plano de cuidados individualizados especialmente na área da Nefrologia.

\section{Agradecimentos}

À fonte de financiamento Coordenação de Aperfeiçoamento de Pessoal Nível Superior - Brasil (CAPES) e às acadêmicas de Enfermagem Julia Faraon Kapitansky e
Julia Domingues de Figueiredo, pelo auxílio na coleta de dados desta pesquisa.

Recibido: 26-03-19

Revisado: 20-05-19

Modificado: 01-06-19

Aceptado: 15-06-19

\section{Bibliografía}

1. Webster CA, Nagler EV, Morton RL, Masson P. Chronic kidney disease. Lancet 2017 mar;389 (10075):1238-52.

2. Pilger C, Rampari EM, Waidman MAP, Carreira L. Hemodiálise: seu significado e impacto para a vida do idoso. Esc. Anna Nery 2010 out-dez;14(4):677-83.

3. Figueiredo $A E$, Bernardini J, Bowes $E$, Hiramatsu $M$, Price V, Su C et al. ISPD guideline/recommendations: a syllabus for teaching peritoneal dialysis to patients and caregivers. Perit Dial Int 2016;36 (6):592-605.

4. Santos CM, Kirchmaier FM, Silveira WJ, Arreguy-Sena C. Percepções de enfermeiros e clientes sobre cuidados de enfermagem no transplante de rim. Acta Paul Enferm 2015;28(4):337-43.

5. Weiss BD, Mays MZ, Martz W, Castro KM, DeWalt DA, Pignone MP et al. Quick assessment of literacy in primary care: the newest vital sign. Annals of Fam Med 2005;3(6):514-22.

6. Santos MIP0, Portella MR, Scortegagna HDM, Santos PCS. Letramento funcional em saúde na perspectiva da Enfermagem Gerontológica: revisão integrativa da literatura. Ver Bras Geriatr Gerontol 2015;18(3):651-64.

7. Santos LTM, Mansur HN, Paiva TFPS, Colugnati FAB, Bastos MG. Letramento em saúde: importância da avaliação em nefrologia. J Bras Nefrol 2012; 34(3):293-302. 
8. Finkelstein F0, Story K, Firanek C, Barre P, Takano $\mathrm{T}$, Soroka $\mathrm{S}$ et al. Perceived knowledge among patients cared for by nephrologists about chronic kidney disease and end-stage renal disease therapies. Kidney Int 2008;74(9):1178-84.

9. Cavanaugh $\mathrm{KL}$, Wingard $\mathrm{RL}$, Hakim RM, Eden $\mathrm{S}$, Shintani A, Wallston KA. Low health literacy associates with increased mortality in ESRD.J Am Soc Neph 2010;21(11):1979-85.

10. Demian MN, Shapiro RJ, Thornton WL. An observational study of health literacy and medication adherence in adult kidney transplant recipients. NDT Plus 2016;9(6):858-65.

11. Taylor DM, Bradley A, Bradley C, Draper H, Dudley C, Fogarty D. Limited health literacy is associated with reduced access to kidney transplantation. Kidney Inter 2019;95(5):1244-52.

12. Guanaré VCSC, Maranho KMP, França AKTC, Cavalcante MCV. Fatores associados à função cognitiva de pacientes com Doença Renal Crônica. Cad Ter Ocup UFSCar 2016;24(2):288-96.

13. Dodson S, Osicka T, Huang L, McMahon LP, Roberts MA. Multifaceted assessment of health literacy in people receiving dialysis: Associations with psychological stress and quality of life. J Health Commun 2016;21(2):91-8.

14. Morisky DE, Green LW, Levine DM. Concurrent and predictive validity of a self-reported measure of medication adherence. Med Care 1986; 24 (1): 67-74.

15. Brucki SMD, Nitrini R, Caramelli P, Bertolucci PHF, Okamoto IH. Sugestão para o uso do mini-exame do estado mental no Brasil. Arq Neuro-Psiquiatr 2003; 61(3):777-81.

16. Melo DM, Barbosa AJG. 0 uso do Mini-Exame do Estado Mental em pesquisas com idosos no Brasil: uma revisão sistemática. Cien Saude Colet 2015; 20:3865-76.

17. Apolinario $D$, Oliveira RC, Bragal $P$, Magaldil RM, Bussel AL, Camporal F et al. Short assessment of health literacy for Portuguese-speaking adults. Rev Saúde Pública 2012;46(4):702-11.
18. Ciconelli RM, Ferraz MB, Santos W, Meinao IM, Quaresma MR. Brazilian-Portuguese version of the SF-36. A reliable and valid quality of life outcome measure. Rev Bras Reumatol 1999;39(3):143-50.

19. Green JA, Mor MK, Shields AM, Sevick MA, Arnold RM, Palevsky PM et al. Associations of health literacy with dialysis adherence and health resource utilization in patients receiving maintenance hemodialysis. Am J Kidney Dis 2013;62(1):73-80.

20. Brice JH, Foster MB, Principe S, Moss C, Shofer FS, Falk RJ et al. Single-item or two-item literacy screener to predict the S-TOFHLA among adult hemodialysis patients. Patient Educ Couns 2014;94 (1):71-5.

21. Adeseun GA, Bonney CC, Rosas SE. Health literacy associated with blood pressure but not other cardiovascular disease risk factors among dialysis patients.J Clin Hypertens 2012;25(3):348-53.

22. Jain D, Sheth H, Green JA, Bender FH, Weisbord SD. Health literacy in patients on maintenance peritoneal dialysis: prevalence and outcomes. Perit Dial Int 2015;35(1):96-8.

23. Lambert K, Mullan J, Mansfield K, Lonergan M. A cross-sectional comparison of health literacy deficits among patients with chronic kidney disease.J Health Commun 2015;20(2):16-23.

24. Kazley AS, Hund JJ, Simpson KN, Chavin K, Baliga P. Health literacy and kidney transplant outcomes. Prog Transplant 2015;25(1):85-90.

25. Dageforde LA, Petersen AW, Feurer ID, Cavanaugh $\mathrm{KL}$, Harms KA, Ehrenfeld JM et al. Health literacy of living kidney donors and kidney transplant recipients. Transplant 2014;98(1):88-93.

26. Yudell $M$, Roberts $D$, DeSalle $R$, Tishkoff $S$. Taking race out of human genetics.Science;2016(351):564-5.

27. Baker DW, Williams MV, Parker RM, Gazmararian JA, Nurss J. Development of a brief test to measure functional health literacy. Patient Educ Couns 1999;38(1):33-42.

28. Sgnaolin V, Figueiredo AEPL. Adesão ao tratamento farmacológico de pacientes em hemodiálise. J. Bras. Nefrol 2012;34(2):109-16. 
29. Batista Alves B, Vital Guilarducci N, dos Reis Santos T, Baldoni A0, Otoni A, Pinto SW et al. Existe associação entre qualidade de vida e adesão à farmacoterapia em pacientes com doença renal crônica em hemodiálise? Einstein 2018;16(1):1-7.

30. Barbosa Moreira L, Camurça Fernandes PFCB, Sueli Monte F, Costa Martins AM. Adesão ao tratamento farmacológico em pacientes com doença renal crônica. J Bras Nefrol 2008;30(2):113-9.

31. Coelho Gonçalves PR, Ferreira Reveles A, Fernandes Martins HI, Lourenço Rodrigues I, Miranda Rodrigues SM. A Adesão à Terapêutica Imunossupressora na Pessoa Transplantada Renal. Rev Enf Ref 2016;IV(8):121-31.
32. Lee $S$, Shimada H, Park H, Makizako H, Lee S, Doi $T$ et al. The association between kidney function and cognitive decline in community-dwelling, elderly Japanese people. J Am Med Dir Assoc 2015;16(4): 349.el-5.

33. Cavanaugh $\mathrm{KL}$, Osborn $\mathrm{CY}$, Tentori $\mathrm{F}$, Rothman $\mathrm{RL}$, Ikizler TA, Wallston KA. Performance of a brief survey to assess health literacy in patients receiving hemodialysis. Clin Kidney J 2015;8(4):462-8.

34. Lima Condé SA, Fernandes N, Rossi dos Santos F, Chouab F, Peruzzi da Mota M ME, Gomes Bastos M. Cognitive decline, depression and quality of life in patients at different stages of chronic kidney disease. J. Bras. Nefrol 2010;32(3):242-8.

Este artículo se distribuye bajo una Licencia Creative Commons Atribución-NoComercial 4.0 Internacional. https://creativecommons.org/licenses/by-nc/4.0/

\section{Open Access (c) ()}

